\title{
Pacific
}

Journal of

Mathematics

\section{CROSSCAP NUMBER OF A KNOT}

\section{Hitoshi MURAKami AND AKIRA YASUhaRa}




\title{
CROSSCAP NUMBER OF A KNOT
}

\author{
Hitoshi Murakami ANd AKIRA Yasuhara
}

\begin{abstract}
B. E. Clark defined the crosscap number of a knot to be the minimum number of the first Betti numbers of non-orientable surfaces bounding it. In this paper, we investigate the crosscap numbers of knots. We show that the crosscap number of $7_{4}$ is equal to 3 . This gives an affirmative answer to a question given by Clark. In general, the crosscap number is not additive under the connected sum. We give a necessary and sufficient condition for the crosscap number to be additive under the connected sum.
\end{abstract}

\section{Introduction.}

We study knots in the 3 -sphere $S^{3}$. The genus $g(K)$ of a knot $K$ is the minimum number of the genera of Seifert surfaces for it [11]. Here a Seifert surface means a connected, orientable surface with boundary $K$. In 1978, B. E. Clark [3] defined the crosscap number $C(K)$ of $K$ to be the minimum number of the first Betti numbers of connected, non-orientable surfaces bounding it. (For the trivial knot, it is defined to be 0 instead of 1.) He proved the following inequality and asked whether there exist knots for which the equality holds.

$$
C(K) \leq 2 g(K)+1 .
$$

Note that since $C$ (trivial knot) $=0$, the equality does not hold for the trivial knot. In this paper, we give an example which satisfies the equality showing that $C\left(7_{4}\right)=3$. (We use the notation of J. W. Alexander and B. G. Briggs for knots [1]. See also [9] and [2].)

Clark also studied how the crosscap number behaves under the connected sum. If we denote by $\Gamma(K)$ the minimum number of the first Betti numbers of connected, unoriented surfaces bounding it (an unoriented surface means a surface which is orientable or not), $\Gamma(K)$ is additive under the connected sum, i.e., $\Gamma\left(K_{1} \sharp K_{2}\right)=\Gamma\left(K_{1}\right)+\Gamma\left(K_{2}\right)[3$, Lemma 2.7] as H. Schubert proved for the genus [10]. Note that $\Gamma(K)=\min (2 g(K), C(K))$. A proof is given by an ordinary "cut-and-paste" argument. See for example [9, Theorem 5A14]. But such an argument does not apply to the crosscap number because one of the two surfaces obtained from a non-orientable surface by cutting along an 


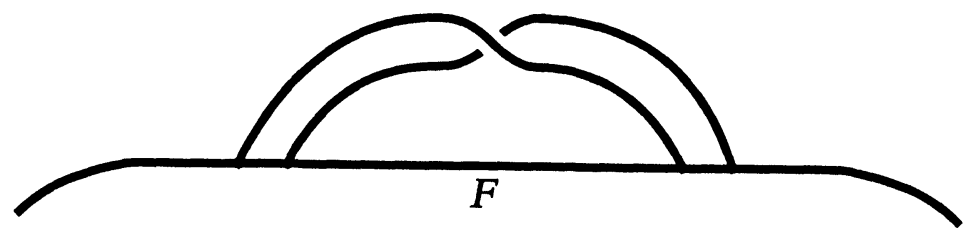

Figure 1.1.

arc may be orientable. But Clark proved that $C\left(K_{1} \sharp K_{2}\right)$ is either $C\left(K_{1}\right)+$ $C\left(K_{2}\right)-1$ or $C\left(K_{1}\right)+C\left(K_{2}\right)$. More precisely, he proved that

$$
C\left(K_{1}\right)+C\left(K_{2}\right)-1 \leq C\left(K_{1} \sharp K_{2}\right) \leq C\left(K_{1}\right)+C\left(K_{2}\right) .
$$

If we put $K_{1}=K_{2}=7_{4}$, we have the first equality and if we put $K_{1}=K_{2}=$ $3_{1}$, we have the second equality (this is not so interesting). More generally, we can show that the second equality holds if and only if $C\left(K_{i}\right)=\Gamma\left(K_{i}\right)$ for $i=1$ and 2 .

\section{Inequalities.}

In this section, we will give some inequalities concerning $\Gamma(K)$ and $C(K)$.

As an upper bound for $C(K)$, Clark proved the following by adding a half-twisted band to a Seifert surface as in Figure 1.1.

Proposition 1.1. (B.E. Clark [3, Proposition 2.6]).

$$
C(K) \leq 2 g(K)+1
$$

Now we give another upper bound in terms of the crossing number. Let $n(K)$ be the minimum crossing number of a knot $K$. Then we have

\section{Proposition 1.2.}

$$
\Gamma(K) \leq\left[\frac{n(K)}{2}\right]
$$

where $[x]$ denotes the greatest integer which does not exceed $x$.

Proof. Let $D$ be a diagram in $S^{2}$ of $K$ with minimum crossings. We colour the regions of $D$ with black and white like a chess-board and denote by $b$ and $w$ the numbers of black regions and white regions respectively. Then we can construct two surfaces bounding $K$ form the black regions and the white regions. So $\Gamma(K)$ is less than or equal to the first Betti number of 
these surfaces. Since the number of all the regions is $n(K)+2$, we have

$$
\max (b, w) \geq \begin{cases}\frac{n(K)+2}{2} & \text { if } n(K) \text { is even, } \\ \frac{n(K)+3}{2} & \text { if } n(K) \text { is odd. }\end{cases}
$$

Now from an argument using the Euler characteristics, we have

$$
\Gamma(K) \leq 1+n(K)-\max (b, w) \leq\left[\frac{n(K)}{2}\right] .
$$

Thus the proof is complete.

For the crosscap number, we have

Proposition 1.3.

$$
C(K) \leq\left[\frac{n(K)}{2}\right]
$$

Proof. If the surface constructed above is non-orientable, the inequality clearly holds. If it is orientable and its first Betti number is strictly less than $[n(K) / 2]$, then we can add a half-twisted band as in Figure 1.1 and make it non-orientable. (This was observed by Clark in the proof of [3, Proposition 2.6].) Since the first Betti number increases by one, the inequality still holds. So we assume without loss of generality that the surface constructed from the black regions (black surface) is orientable and its first Betti number is equal to $[n(K) / 2]$.

If $n(K) \equiv 2$ or $3 \bmod 4$, then $[n(K) / 2]$ is odd. But this cannot occur since a surface with odd first Betti number must be non-orientable.

Next we consider the case that $n(K) \equiv 0 \bmod 4$. Since the first Betti number of the white surface is also $[n(K) / 2]=n(K) / 2$ in this case, the result follows since the white surface must be non-orientable (if $n(K) \neq 0$ ) as indicated in Figure 1.2 and so we can choose the white one. (It was observed by Clark in the proof of Theorem 2.1 in [3] that either black or white surface is non-orientable.) If $n(K)=0$, then $K$ is the trivial knot and the equality holds from the definition.

Finally we consider the case that $n(K) \equiv 1 \bmod 4$. Let $b$ and $w$ be the numbers of black regions and white regions respectively, and $B$ and $W$ the black and the white surfaces respectively. From the assumption, $\beta_{1}(B)=[n(K) / 2]=(n(K)-1) / 2$ and so we have $b=(n(K)+3) / 2$ since $\beta_{1}(B)=1+n(K)-b$, where $\beta_{1}$ denotes the first Betti number. Since $b+w=n(K)+2$, we have

$$
n(K)=2 w-1
$$



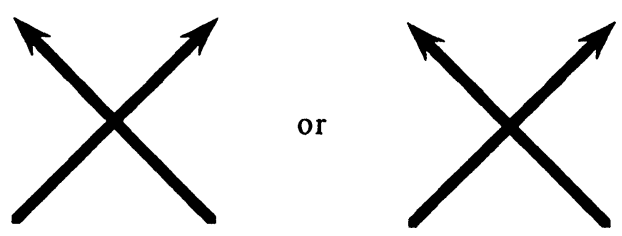

Figure 1.2.

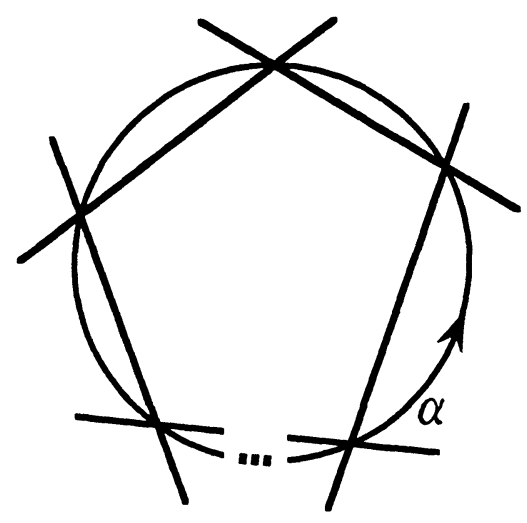

Figure 1.3.

Now we see that the number of edges of every white region is even, since otherwise an arc $\alpha$ indicated in Figure 1.3 is non-orientable and so is $B$.

Let $2 x_{1}, 2 x_{2}, \ldots$, and $2 x_{w}$ be the numbers of edges of white regions. Since the number of all the edges is $2 n(K)$, we have

$$
n(K)=\sum_{i=1}^{w} x_{i}
$$

Since $n(K)=2 w-1$, we see there exists a white region which has only two edges. If we replace a neighbourhood of this region as in Figure 1.4, we have a non-orientable surface $B^{\prime}$ with the same first Betti number. So we have $C(K) \leq \beta_{1}\left(B^{\prime}\right)=\beta_{1}(B)=[n(K) / 2]$.

So the proof is complete.

Observation 1.4. The inequalities in Propositions 1.2 and 1.3 are best possible if $n(K) \equiv 0,1$, or $3 \bmod 4$, i.e., we have examples satisfying the equalities in these cases.

Proof. If $n(K)=4 m$ for some integer $m \geq 0$, we take $\sharp_{m} 4_{1}$, where $\sharp_{m} 4_{1}$ is the connected sum of $m$ copies of $4_{1}$. Since $\sharp_{m} 4_{1}$ is alternating, we have 

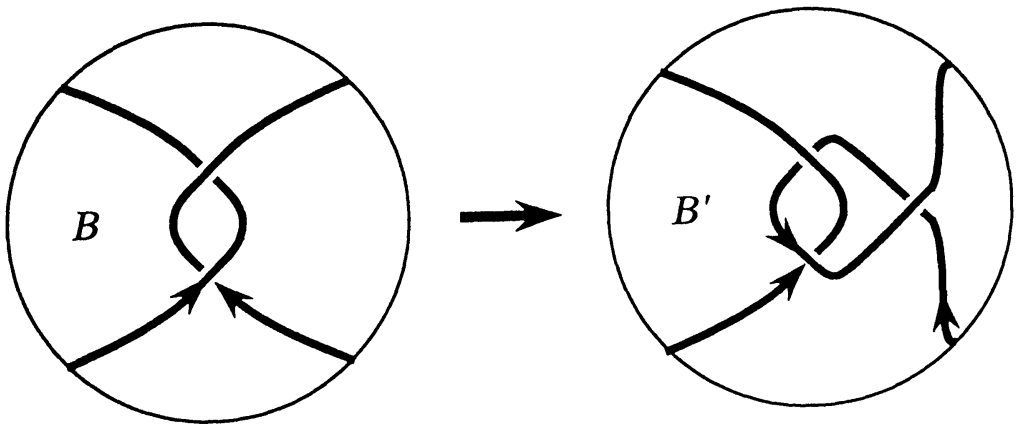

Figure 1.4.

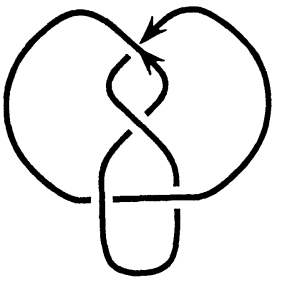

(a)

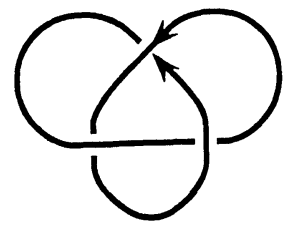

(c)

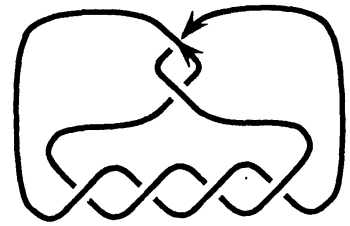

(b)

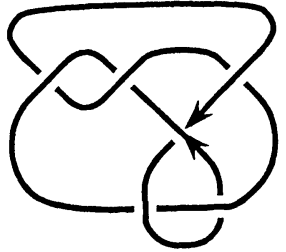

(d)

Figure 1.5.

$n\left(\sharp_{m} 4_{1}\right)=4 m$ from Tait's conjecture [5], [7], [12]. As indicated in Figure 1.5(a), $C\left(4_{1}\right) \leq 2$. From Proposition 2.1 , we see that $C\left(4_{1}\right)=2$ since $4_{1}$ is not a cable knot. So we have $\Gamma\left(4_{1}\right)=\min \left(2 g\left(4_{1}\right), C\left(4_{1}\right)\right)=2$. Thus $C\left(\sharp_{m} 4_{1}\right)=\Gamma\left(\sharp_{m} 4_{1}\right)=2 m=[4 m / 2]$ from the additivity of $\Gamma(K)$. So the equalities in Propositions 1.2 and 1.3 follow in this case.

If $n(K)=4 m+1(m \geq 1)$, then we take $5_{2} \sharp\left(\sharp_{m-1} 4_{1}\right)$. $n\left(5_{2} \sharp\left(\sharp_{m-1}\right.\right.$ $\left.4_{1}\right)$ ) is equal to $4 m+1$ as above. Since $\Gamma\left(5_{2}\right)=C\left(5_{2}\right)=2$ from Figure 1.5(b) and Proposition 2.1, we see that $C\left(5_{2} \sharp\left(\sharp_{m-1} 4_{1}\right)\right)=\Gamma\left(5_{2} \sharp\left(\sharp_{m-1} 4_{1}\right)\right)=$ $2 m=[(4 m+1) / 2]$ by the same reason as above. This gives the equalities for $n(K)=4 m+1$.

If $n(K)=4 m+3(m \geq 0)$, we take $3_{1} \sharp\left(\sharp_{m} 4_{1}\right)$. Since $\Gamma\left(3_{1}\right)=C\left(3_{1}\right)=1$ (see Figure 1.5(c)), we have $C\left(3_{1} \sharp\left(\sharp_{m} 4_{1}\right)\right)=\Gamma\left(3_{1} \sharp\left(\sharp_{m} 4_{1}\right)\right)=2 m+1=[(4 m+$ 
3)/2], and the proof is complete.

Remark 1.5. As shown in Figure 1.5(d), $\Gamma\left(6_{3}\right)=C\left(6_{3}\right) \leq 3$. (Note that $g\left(6_{3}\right)=2$.) But the authors do not know whether the equality holds or not. If it holds, then the equalities in Propositions 1.1 and 1.2 also hold for $n(K) \equiv 2 \bmod 4$.

Let $D(K)$ be the double branched cover of $S^{3}$ branched along a knot $K$ and $e_{2}(K)$ be the minimum number of generators of $H_{1}(D(K) ; \mathbb{Z})$. Then for a lower bound, we have

Proposition 1.6. $\Gamma(K) \geq e_{2}(K)$. Thus we have $C(K) \geq e_{2}(K)$ and $2 g(K) \geq e_{2}(K)$.

Proof. Since we can construct the double branched cover of $K$ by cutting along an unoriented surface bounding it, we have $\Gamma(K) \geq e_{2}(K)$. The second and the third inequalities follow from the definition of $\Gamma(K)$.

\section{Knots with crosscap number two.}

If a knot bounds a Möbius band, it is a cable knot of the centre line of the band. So we have the following proposition due to Clark [3, Proposition 2.2].

Proposition 2.1. (B.E. Clark). A non-trivial knot has crosscap number one if and only if it is a $(2, p)$-cable of some knot for an odd integer $p$.

Now consider knots with crosscap number two.

For an unoriented surface $F$ with boundary a knot $K, \mathrm{C}$. McA. Gordon and R. A. Litherland define a Goeritz matrix $G_{F}(K)$ as follows [4]. Choose a generator system $\left\{\alpha_{1}, \alpha_{2}, \ldots, \alpha_{d}\right\}$ for $H_{1}(F ; \mathbb{Z})$. Then the $(i, j)$-entry of $G_{F}(K)$ is defined to be $\operatorname{lk}\left(a_{\imath}, \tau a_{j}\right)$, where $\mathrm{lk}$ is the linking number, $a_{i}$ is a 1-cycle representing $\alpha_{i}$, and $\tau a_{j}$ is a 1-cycle in $S^{3}-F$ obtained by pushing off $2 a_{j}$.

The normal Euler number $e(F)$ of $F$ is defined to be $-l k\left(K, K^{\prime}\right)$, where $K^{\prime}$ is the intersection of $F$ and the boundary of the regular neighbourhood of $K$ in $S^{3}$ with orientation parallel to that of $K$.

Now a result of Gordon and Litherland is as follows [4, Corollary 5].

$$
(*) \quad \sigma(K)=\operatorname{sign}\left(G_{F}(K)\right)+\frac{1}{2} e(F),
$$

where $\sigma(K)$ is the signature of $K$ and $\operatorname{sign}\left(G_{F}(K)\right)$ is the signature of the symmetric matrix $G_{F}(K)$. Note that $\sigma(K)$ is by definition equal to $\operatorname{sign}\left(G_{F}(K)\right)$ for orientable surface $F$. We also note that a Goeritz matrix is uniquely determined if one fixes a generator system for $H_{1}(F ; \mathbb{Z})$. 


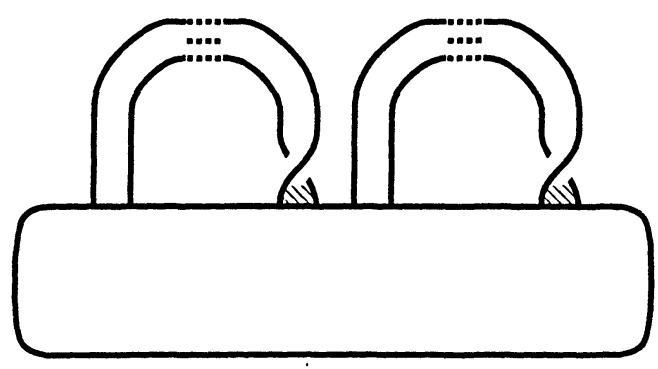

Figure 2.1.

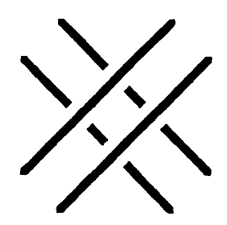

Figure 2.2.

Now we will prove

Theorem 2.2. Suppose that a knot $K$ bounds a non-orientable surface $F$ with the first Betti number two. Then one can choose a generator system for $H_{1}(F ; \mathbb{Z})$ so that the Goeritz matrix $G_{F}(K)$ corresponding to it is of the following form.

$$
G_{F}(K)=\left(\begin{array}{cc}
l & m \\
m & n
\end{array}\right)
$$

with $\sigma(K)=\operatorname{sign}\left(G_{F}(K)\right)-(l+2 m+n)$ for some odd integers $l$ and $n$ and some even integer $m$. Note that $l+2 m+n$ is the sum of all the entries in $G_{F}(K)$.

Proof. We may assume that $F$ is a disc with two non-orientable bands as indicated in Figure 2.1. We also assume that crossings of bands are as in Figure 2.2, except near their heads. Figure 2.3 shows an example.

Choosing a generator system $\{\alpha, \beta\}$ as in figure 2.4 , we see that the Goeritz matrix corresponding to it is

$$
\left(\begin{array}{l}
\operatorname{lk}(a, \tau a) \operatorname{lk}(a, \tau b) \\
\operatorname{lk}(b, \tau a) \operatorname{lk}(b, \tau b)
\end{array}\right)=\left(\begin{array}{cc}
2 w(A, A)+1 & w(A, B) \\
w(B, A) & 2 w(B, B)+1
\end{array}\right) .
$$

Here $a$ and $b$ are 1-cycles representing $\alpha$ and $\beta$ respectively, $A$ and $B$ are bands $\alpha$ and $\beta$ pass through respectively, and $w(X, Y)$ is the sum of the signs of crossings of bands $X$ and $Y$ with signs determined as in Figure 2.5. 


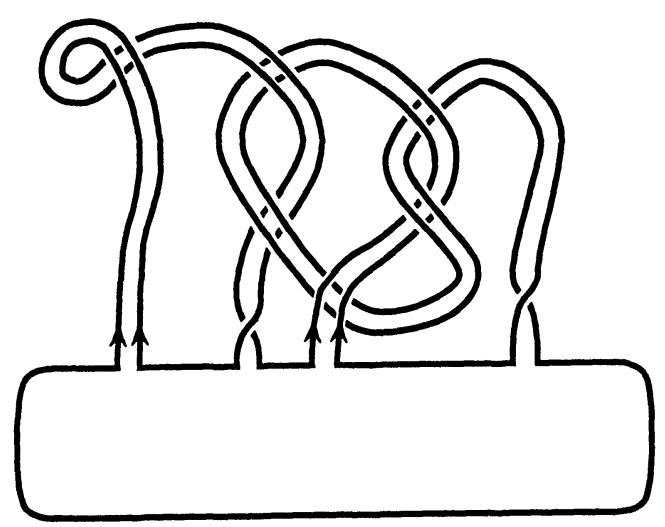

Figure 2.3.

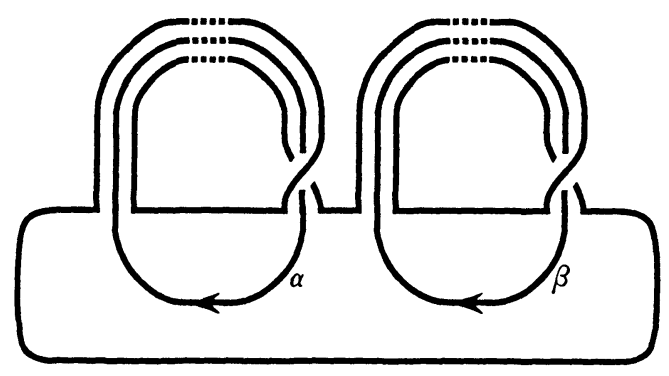

Figure 2.4.

Note that $\operatorname{lk}(a, \tau b)=\operatorname{lk}(b, \tau a)=2 \operatorname{lk}(a, b)$, so $w(A, B)=w(B, A)$ and both $w(A, B)$ and $w(B, A)$ are even.

We also see that the normal Euler number of $F$ is $-2\{2 w(A, A)+w(A, B)+$ $w(B, A)+2 w(B, B)\}-4$ (the last 4 comes from single crossings near the heads of the bands).

From (*), we have

$$
\begin{aligned}
\sigma(K)= & \operatorname{sign}\left(G_{F}(K)\right) \\
& -\{2 w(A, A)+w(A, B)+w(B, A)+2 w(B, B)\}-2 .
\end{aligned}
$$

Thus the proof is complete putting $l=2 w(A, A)+1, n=2 w(B, B)+1$, and $m=w(A, B)=w(B, A)$.

Similarly we can prove

Theorem 2.3. If $C(K) \leq d$, then we can choose a $d \times d$ Goeritz matrix of the following form.

$$
G_{F}(K)=\left(a_{i j}\right)
$$



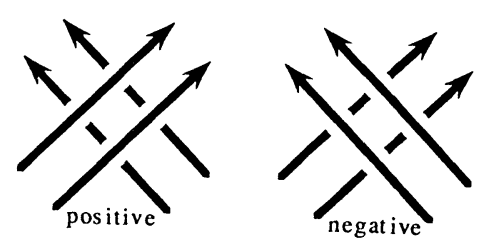

Figure 2.5.

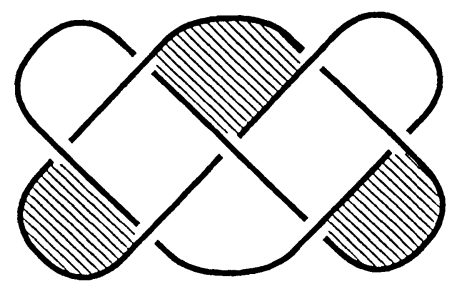

Figure 3.1.

with $a_{i i}$ odd, $a_{i j}$ even $(i \neq j)$, and $\sigma(K)=\operatorname{sign}\left(G_{F}(K)\right)-\sum_{i, j=1}^{d} a_{i j}$.

\section{Crosscap number of $7_{4}$.}

In this section we will prove

Theorem 3.1. $C\left(7_{4}\right)=3$.

As is well known and shown in Figure 3.1, we have $g\left(7_{4}\right)=1$. Thus $C\left(7_{4}\right) \leq 3$ from Proposition 1.1. So we need to prove $C\left(7_{4}\right)>2$.

Note that Proposition 1.6 is not useful at all, since it does not take orientability into account.

Proof of Theorem 3.1. Suppose that $C\left(7_{4}\right) \leq 2$. Then there exists a nonorientable surface $F$ with $\partial F=7_{4}$ and $H_{1}(F ; \mathbb{Z}) \cong \mathbb{Z} \oplus \mathbb{Z}$. The Goeritz matrix $G_{F}\left(7_{4}\right)$ determined by a generator system of $H_{1}(F ; \mathbb{Z})$ is a $2 \times 2$ matrix. If one changes a generator system by using a unimodular matrix $P$, then a Goeritz matrix becomes $P^{t} G_{F}\left(7_{4}\right) P$, where $P^{t}$ is the transposed matrix of $P$. Since the absolute value of the determinant of a Goeritz matrix of a knot is equal to the order of $H_{1}\left(D\left(7_{4}\right) ; \mathbb{Z}\right)$, the determinant of $G_{F}\left(7_{4}\right)$ is \pm 15 . From an elementary theory of binary quadratic forms (see for example $[8,3.5])$, there are the following seven cases to be considered after changes 
of generator systems.

$$
\begin{aligned}
& \text { (i) } \varepsilon\left(\begin{array}{cc}
1 & 0 \\
0 & 15
\end{array}\right), \quad \text { (ii) } \varepsilon\left(\begin{array}{ll}
2 & 1 \\
1 & 8
\end{array}\right), \quad \text { (iii) } \varepsilon\left(\begin{array}{ll}
3 & 0 \\
0 & 5
\end{array}\right), \quad \text { (iv) } \varepsilon\left(\begin{array}{ll}
4 & 1 \\
1 & 4
\end{array}\right), \\
& \text { (v) } \varepsilon\left(\begin{array}{cc}
3 & 0 \\
0 & -5
\end{array}\right), \quad\left(\text { vi) } \varepsilon\left(\begin{array}{cc}
2 & 1 \\
1 & -7
\end{array}\right), \quad(\text { vii }) \varepsilon\left(\begin{array}{cc}
1 & 0 \\
0 & -15
\end{array}\right),\right.
\end{aligned}
$$

with $\varepsilon= \pm 1$.

First of all, we consider the linking form $\lambda: H_{1}\left(D\left(7_{4}\right) ; \mathbb{Z}\right) \times H_{1}\left(D\left(7_{4}\right) ; \mathbb{Z}\right) \rightarrow$ $\mathbb{Q} / \mathbb{Z}$. (For the definition and the way to calculate it from the Goeritz matrix, see [4]. ) Since the Goeritz matrix corresponding to the oriented Seifert surface described in Figure 3.1 is $\left(\begin{array}{cc}-4 & 1 \\ 1 & -4\end{array}\right)$ with appropriate generator system, there is a generator $g$ of $H_{1}\left(D\left(7_{4}\right) ; \mathbb{Z}\right)(\cong \mathbb{Z} / 15 \mathbb{Z})$ such that $\lambda(g, g)=-4 / 15$ in $\mathbb{Q} / \mathbb{Z}$. From an easy calculation, we see that the cases (i) with $\varepsilon=-1$, (iv) with $\varepsilon=-1$, and (vii) with $\varepsilon=1$ are possible. Thus we only need to consider

$$
X=\left(\begin{array}{cc}
-1 & 0 \\
0 & -15
\end{array}\right), \quad Y=\left(\begin{array}{ll}
-4-1 \\
-1-4
\end{array}\right), \quad \text { and } Z=\left(\begin{array}{cc}
1 & 0 \\
0 & -15
\end{array}\right)
$$

Now we use Theorem 2.2. Since $\sigma\left(7_{4}\right)=-2$, there exists an integral matrix $Q=\left(\begin{array}{cc}p & q \\ r & s\end{array}\right)$ with $p s-q r= \pm 1$ such that the diagonal entries of $Q^{t} M Q$ is odd and that

$$
(* *) \operatorname{sign}(M)-\left(\text { the sum of all the entries in } Q^{t} M Q\right)=-2,
$$

where $M$ is $X, Y$, or $Z$.

We note that the diagonal entries of $Q^{t} Y Q$ are even. This contradicts the fact that the diagonal entries of $Q^{t} Y Q$ must be odd. (Here the equation $(* *)$ is not used.)

Since the sum of all the entries in $Q^{t} X Q$ is equal to $-(p+q)^{2}-15(r+s)^{2}$, we have

$$
-2+(p+q)^{2}+15(r+s)^{2}=-2
$$

from $(* *)$. But the solution to this equation is $p+q=r+s=0$, which is impossible since $Q$ must be unimodular.

The sum of all entries in $Q^{t} Z Q$ is equal to $(p+q)^{2}-15(r+s)^{2}$. So we have

$$
(p+q)^{2}-15(r+s)^{2}=2 .
$$

But this is also impossible since the equation becomes

$$
(p+q)^{2} \equiv 2 \bmod 3
$$


Thus the proof is complete.

Remark 3.2. As W. B. R. Lickorish mentioned in [6], $7_{4}$ bounds a Möbius band in a 4 -ball. So we cannot prove Theorem 3.1 by using a 4 -dimensional technique.

Remark 3.3. We cannot prove that $C\left(6_{3}\right)>2$ using Theorem 2.2, since the matrix $\left(\begin{array}{cc}7 & -6 \\ -6 & 7\end{array}\right)$ satisfies the condition of the theorem and the linking form determined by it coincides with that of $6_{3}$.

\section{Behaviour under the connected sum.}

Clark studied the behaviour of the crosscap number under the connected sum and show the following inequality.

Proposition 4.1. (B.E. Clark [3, Theorem 2.8]).

$$
C\left(K_{1}\right)+C\left(K_{2}\right)-1 \leq C\left(K_{1} \sharp K_{2}\right) \leq C\left(K_{1}\right)+C\left(K_{2}\right) .
$$

We will prove this proposition carefully to obtain a necessary and sufficient condition for the equalities (Proposition 4.3). Our proof is essentially the same as Clark's. Before proving this, we prepare a lemma.

\section{Lemma 4.2.}

$$
C\left(K_{1} \sharp K_{2}\right)=\min \left(C\left(K_{1}\right)+\Gamma\left(K_{2}\right), \Gamma\left(K_{1}\right)+C\left(K_{2}\right)\right) .
$$

Proof. It is easy to see

$$
C\left(K_{1} \sharp K_{2}\right) \leq \min \left(C\left(K_{1}\right)+\Gamma\left(K_{2}\right), \Gamma\left(K_{1}\right)+C\left(K_{2}\right)\right) .
$$

So we prove

$$
C\left(K_{1} \sharp K_{2}\right) \geq \min \left(C\left(K_{1}\right)+\Gamma\left(K_{2}\right), \Gamma\left(K_{1}\right)+C\left(K_{2}\right)\right) .
$$

Let $F$ be a non-orientable surface bounding $K_{1} \sharp K_{2}$ with $\beta_{1}(F)=C\left(K_{1} \sharp K_{2}\right)$. Let $S$ be a 2 -sphere separating the two connected summands. We may assume that the intersection of $S$ and $F$ is the disjoint union of simple loops and an arc. Let $D$ be a disc bounding an innermost loop in $S$ with no loop or arc in it.

We see that $\partial D$ cuts $F$ into two connected components. For otherwise the (unoriented) surface $F^{\prime}$ obtained from "cut-and-paste" along $D$ has the first Betti number $\beta_{1}(F)-2$. On the other hand, we can construct from $F^{\prime \prime}$ a non-orientable surface with the first Betti number $\beta_{1}(F)-1$ adding a twisted band as in Figure 1.1 if necessary, which contradicts to the minimality of $F$. 
So we have a surface $F^{\prime}$ with boundary $K_{1} \sharp K_{2}$ and a closed surface $F^{\prime \prime}$ after "cut-and-paste" along $D$. From the minimality, $F$ " is a sphere and so $F^{\prime}$ is non-orientable.

Continuing these processes, we can construct a non-orientable surface which intersects $S$ only in an arc.

Thus we have two unoriented surfaces $F_{1}$ and $F_{2}$ bounding $K_{1}$ and $K_{2}$ respectively with $\beta_{1}\left(F_{1}\right)+\beta_{1}\left(F_{2}\right)=\beta_{1}(F)$. Since $F$ is a boundary-connectedsum of $F_{1}$ and $F_{2}$, either $F_{1}$ or $F_{2}$ is non-orientable.

If $F_{1}$ is non-orientable, then we have $C\left(K_{1}\right) \leq \beta_{1}\left(F_{1}\right)$ and $\Gamma\left(K_{2}\right) \leq \beta_{1}\left(F_{2}\right)$. Thus $C\left(K_{1} \sharp K_{2}\right)=\beta_{1}(F)=\beta_{1}\left(F_{1}\right)+\beta_{2}\left(F_{2}\right) \geq C\left(K_{1}\right)+\Gamma\left(K_{2}\right)$. If $F_{2}$ is nonorientable, we have $C\left(K_{1} \sharp K_{2}\right) \geq \Gamma\left(K_{1}\right)+C\left(K_{2}\right)$. So we have the required inequality and the proof is complete.

Proof of Proposition 4.1. Since $\Gamma(K)=\min (2 g(K), C(K))$ and $C(K) \leq$ $2 g(K)+1$ from Proposition 1.1, we have $C(K)-\Gamma(K) \leq 1$. So $C\left(K_{1}\right)+$ $\Gamma\left(K_{2}\right) \geq C\left(K_{1}\right)+C\left(K_{2}\right)-1$ and $\Gamma\left(K_{1}\right)+C\left(K_{2}\right) \geq C\left(K_{1}\right)+C\left(K_{2}\right)-1$. Therefore we have the required formula from the previous lemma.

Now we see that $C\left(K_{1} \sharp K_{2}\right)$ is equal to $C\left(K_{1}\right)+C\left(K_{2}\right)-1$ or $C\left(K_{1}\right)+$ $C\left(K_{2}\right)$. Since $\Gamma(K) \leq C(K)$, we have from Lemma 4.2 a necessary and sufficient condition to decide which value it takes.

Proposition 4.3. $C\left(K_{1} \sharp K_{2}\right)=C\left(K_{1}\right)+C\left(K_{2}\right)$ if and only if $C\left(K_{1}\right)=$ $\Gamma\left(K_{1}\right)$ and $C\left(K_{2}\right)=\Gamma\left(K_{2}\right)$.

Since $C\left(7_{4}\right)=3>\Gamma\left(7_{4}\right)=2$, we have

\section{Corollary 4.4.}

$$
C\left(K \sharp 7_{4}\right)=C(K)+C\left(7_{4}\right)-1 .
$$

In particular, we see $C\left(7_{4} \sharp 7_{4}\right)=5$.

Since $g(K) \geq 1$ for a non-trivial knot $K$, we have

Corollary 4.5. For any non-trivial knots $K_{1}, K_{2}, \ldots, K_{n}$ with $C\left(K_{i}\right)_{2} \leq$ $2(i=1,2, \ldots, n)$, we have

$$
C\left(K_{1} \sharp K_{2} \sharp \ldots \sharp K_{n}\right)=C\left(K_{1}\right)+C\left(K_{2}\right)+\ldots+C\left(K_{n}\right) .
$$




\section{Acknowledgments.}

This paper was written when the first author was visiting the Department of Pure Mathematics and Mathematical Statistics, University of Cambridge. He thanks Dr. W. B. R. Lickorish for his kindness.

\section{References}

[1] J.W. Alexander and G.B. Briggs, On types of knotted curves, Ann. of Math., (2) 28 (1927), 562-586.

[2] G. Burde and H. Zieschang, Knots, de Gruyter Studies in Mathematics; 5, Walter de Gruyter, Berlin, New York, 1985.

[3] B.E. Clark, Crosscaps and knots, Internat. J. Math. and Math. Sci., 1 (1978), 113-123.

[4] C.McA. Gordon and R.A. Litherland, On the signature of a link, Invent. Math., 47 (1978), 53-69.

[5] L.H. Kauffman, State models and the Jones polynomial, Topology, 26 (1987), 395407.

[6] W.B.R. Lickorish, Unknotting by adding a twisted band, Bull. London Math. Soc., 18 (1986), 613-615.

[7] K. Murasugi, Jones polynomial and classical conjectures in knot theory, Topology, 26 (1987), 187-194.

[8] I. Niven, H.S. Zuckerman, and H.L. Montgomery, An Introduction to the Theory of Numbers (Fifth Edition), John Wiley \& Sons, Inc., New York, Chichester, Brisbane, Toronto, Singapore, 1991.

[9] D. Rolfsen, Knots and Links, Publish or Perish, Inc., Berkeley, 1975.

[10] H. Schubert, Die eindeutige Zerlegbarkeit eines Knoten in Primknoten, S. -B. Heidelberger Akad. Wiss. Math.-Nat. Kl., 3 (1949), 57-104.

[11] H. Seifert, Über das Geschlecht von Knoten, Math. Ann., 110 (1934), 571-592.

[12] M.B. Thistlethwaite, A spanning tree expantion of the Jones polynomial, Topology, 26 (1987), 297-309.

Received October 22, 1992 and in revised form February 8, 1993.

Osaka City University

SUgIMOTO, SUMIYOSHI-KU

OSAKA 558, JAPAN

AND

TOKYo Denki UNIVERSity

Hatoyama-MAChi, Saitama-ken

350-03, JAPAN 



\title{
PACIFIC JOURNAL OF MATHEMATICS
}

Founded by E. F. Beckenbach (1906-1982) and F. Wolf (1904-1989)

\section{EDITORS}

\author{
Sun-Yung Alice Chang (Managing Editor) \\ University of California. \\ Los Angeles, CA 90095-1555 \\ pacific@math.ucla.edu
}

\section{F. Michael Christ}

University of California

Los Angeles, CA 90095-1555

christ@math.ucla.edu

Thomas Enright

University of California

San Diego, La Jolla, CA 92093

tenright@ucsd.edu

Nicholas Ercolani

University of Arizona

Tucson, AZ 85721

ercolani@math.arizona.edu
Robert Finn

Stanford University

Stanford, CA 94305

finn@gauss.stanford.edu

Vaughan F. R. Jones

University of California

Berkeley, CA 94720

vfr@math.berkeley.edu

Steven Kerckhoff

Stanford University

Stanford, CA 94305

spk@gauss.stanford.edu
Martin Scharlemann

University of California

Santa Barbara, CA 93106

mgscharl@math.ucsb.edu

Gang Tian

Courant Institute

New York University

New York, NY 10012-1100

tiang@taotao.cims.nyu.edu

\section{S. Varadarajan}

University of California

Los Angeles, CA 90095-1555

vsv@math.ucla.edu

\section{SUPPORTING INSTITUTIONS}

\section{CALIFORNIA INSTITUTE OF TECHNOLOGY NEW MEXICO STATE UNIVERSITY \\ OREGON STATE UNIVERSITY \\ STANFORD UNIVERSITY \\ UNIVERSITY OF ARIZONA \\ UNIVERSITY OF BRITISH COLUMBIA \\ UNIVERSITY OF CALIFORNIA UNIVERSITY OF HAWAII}

\author{
UNIVERSITY OF MONTANA \\ UNIVERSITY OF NEVADA, RENO \\ UNIVERSITY OF OREGON \\ UNIVERSITY OF SOUTHERN CALIFORNIA \\ UNIVERSITY OF UTAH \\ UNIVERSITY OF WASHINGTON \\ WASHINGTON STATE UNIVERSITY
}

The supporting Institutions listed above contribute to the cost of publication of this Journal, but they are not owners or publishers and have no responsibility for its contents or policies.

Manuscripts must be prepared in accordance with the instructions provided on the inside back cover.

The Pacific Journal of Mathematics (ISSN 0030-8730) is published monthly except for July and August. Regular subscription rate: $\$ 215.00$ a year (10 issues). Special rate: $\$ 108.00$ a year to individual members of supporting institutions.

Subscriptions, orders for back issues published within the last three years, and changes of subscribers address should be sent to Pacific Journal of Mathematics, P.O. Box 4163, Berkeley, CA 94704-0163, U.S.A. Prior back issues are obtainable from Kraus Periodicals Co., Route 100, Millwood, NY 10546.

The Pacific Journal of Mathematics at the University of California, c/o Department of Mathematics, 981 Evans Hall, Berkeley, CA 94720 (ISSN 0030-8730) is published monthly except for July and August. Second-class postage paid at Berkeley, CA 94704, and additional mailing offices. POSTMASTER: send address changes to Pacific Journal of Mathematics, P.O. Box 6143, Berkeley, CA 94704-0163.

\author{
PUBLISHED BY PACIFIC JOURNAL OF MATHEMATICS at University of California, \\ Berkeley, CA 94720, A NON-PROFIT CORPORATION \\ This publication was typeset using AMS-LATEX, \\ the American Mathematical Society's TEX macro system. \\ Copyright (C) 1995 by Pacific Journal of Mathematics
}




\section{PACIFIC JOURNAL OF MATHEMATICS}

Volume $171 \quad$ No. $1 \quad$ November 1995

Generalized fixed-point algebras of certain actions on crossed products

BEATRIZ ABADIE

Partitions, vertex operator constructions and multi-component KP

equations

MAarten Bergvelt and A. P. E. Ten Kroode

Holomorphy tests based on Cauchy's integral formula

CARMEN CASCANTE and DANIEL PASCUAS

The Euler characteristic of a nonpositively curved, piecewise Euclidean manifold

RUTH CHARNEY and MiChaEl WALTER DAVIS

The invariant connection of a $\frac{1}{2}$-pinched Anosov diffeomorphism and rigidity

RENATO FERES

The inverse Riemann mapping theorem for relative circle domains

ZHENG-XU HE and OdED SCHRAMM

Multipliers and Bourgain algebras of $H^{\infty}+C$ on the polydisk

KeI Ji IZUCHI and YASOU MATSUGU

Irreducible bimodules associated with crossed product algebras. II

TSUYOSHI KAJIWARA and SHIGERU YAMAGAMI

Elliptic fibrations on quartic $K 3$ surfaces with large Picard numbers

MASATO KUWATA

Automatic continuity for weakly decomposable operators

RIDGLEY LANGE, SHENG-WANG WANG and YONG ZHONG

Crosscap number of a knot

Hitoshi MURAKAMI and AKIRA YASUHARA

Symmetric minimal surfaces in $\mathbb{R}^{3}$ 